\author{
Military Technical College \\ Kobry Elkobbah, \\ Cairo, Egypt
}

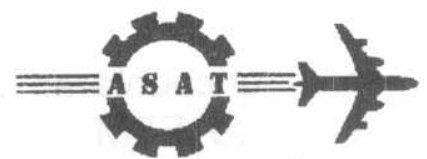

$8^{\text {th }}$ International Conference on Aerospace Sciences \& Aviation Technology

\title{
AUTOMATIC DEINTERLEAVING OF INTERCEPTED RADAR PULSES AT THE INPUT OF ESM SYSTEM
}

\author{
M.H. EI-AYADI", K.A. EI-BARBARY**, H.E. ABOU-BAKR**
}

\begin{abstract}
ABSTR:ACT
A radar Electronic Support Measures (ESM) system performs two functions, warning of impending emitters and surveillance to determine the radar activities. Automatic radar ESM system consists of a passive radar receiver which receives emissicins from other platforms, measures the parameters of each received pulse and a deinterleaver that sorts the intercepted pulses to enable determination of the individual radar parameters. These parameters are compared with the stored parameters of known radars to identify the intercepted emitter type. Consequently the threat evel ano the optimum jamming program for the intercepted emitter could be derived. This paper concentrates on emitters deinterleaving in case of high data rate of about $10^{6}$ pulse/second. Emitter deinterleaving involves sorting and segregating the received radar pulses into separate cells each cell is associated with a single emitter Additional parameters such as PRI, and antenna scan pattern could be evaluated for each cell. A proposed deinterleaving algorithm, which depends on the mono-pulse parameters, is presented. Both the physical and analytic behaviors of this algorithm are examined through computer simulations.
\end{abstract}

\section{KEY WORDS}

Radar pulses, deinterleaving algorithms, sorting and radar cells

\section{NOMEIVCLATURE}

AOA: Angle of arrival

PA : Pulse amplitude

PRI: Pulse repetition Interval

RF : Radio frequency
EDV: Emitter descriptor vector

PDV: Pulse descriptor vector

PW: Pulse width

TOA :Time of arrival

\footnotetext{
* Professsor, Faculty of Computer and Information, Ain Shams University, Cairo, Egypt

** Egyrtian Armed Forces
} 


\section{INTRODUCTION}

In dense electromagnetic environments encountered in modern warfare, the large number of independent emitters will cause ESM system to receive a seemingly random pulse stream consisting of interleaved pulse chains. In order to identify individual emitters, ESM system has relied on operator interpretation of the output of the ESM receiver to achieve this, but now some form of automatic ESM processing is often required to manipulate the high received pulse rates and provide a real time response.

The front-end of an automatic ESM system is designed to produce a digital word representing the parameters of each detectable radar pulse. The deinterleaving processor operates on this mono pulse data and attempt to identify and separate pulse cell due to individual emitters. Ideally there will be a one- to-one correspondence between each pulse cells identified by the deinterleaving process and an emitter in the environments. In order to provide optimum performance, the design of the deinterleaving processor must take into account the expected characteristics of the radar environments, the accuracy and resolution of the measurements systems and all sources of data corruption. In this paper, it will be a proposed automatic deinterleaving algorithm depends on the measured parameters of intercepted stream of radar pulses and their associated root mean square errors. We complete the analysis of the performance of the deinterleaver processor, through computer simulations. The evaluation of the proposed automatic deinterleaving algorithm permits estimating the probability of pulse loss (blocking probability) due to high arrival rates of intercepted radar pulses or due to the limiting processing speed of the deinterleaver processor. The analysis also indicates some system structure trade offs as well as some peculiarities of the performance of the proposed automatic deinterleaving algorithm.

\section{BASIC COMPONENTS OF AUTOMATIC ESM SYSTEM}

Broadly speaking, an automatic ESM system consists of a three main subsystems[2] as shown in Fig.1. The receiver subsystem, which is a passive radar receiver, picks up the pulses transmitted by various radars in the environment and measures their individual parameters. These parameters are angle of arrival (AOA), radio frequency $(R F)$, pulse width (PW), pulse amplitude(PA) and time of arrival (TOA). The receiver is designed to cover wide parameter ranges to ensure detection of all radars of interest. The measured parameters of every successfully intercepted pulse are encoded in digital format called pulse descriptor vector (PDV), which takes the form shown in Fig. 2.

The deinterleaver sorts the PDVs and forms pulse cells, each comprises a set of PDVs assumed to belong to same emitter. Then, each pulse cell is encoded as emitter descriptor vector EDVs whose components are emitter characteristics such as AOA, RF, PW, pulse repetition interval (PRI), agility and antenna scan period. Obviously the better the resolution and accuracy of pulse parameters measurements, the less error the deinterleaver commits in the sorting process and the large number of bits represents this parameter.

The main processor compares estimated EDVs with the stored data in the threat library of EW systern in order to identify the intercepted radar type. In some 
advanced ESM systems, the main processor can further, form identified radar type, instantaneous position data supplied from airborne navigator, angle of arrival information, and electronic order of battle [1] stored in the threat library of the EW system, determine the location of the detected radar emitter. The output of the ESM system is fed to an ECM resource management system, which starts an appropriate ECM action through the data processor and the executive controller to the ECM portion of an integrated EW system.

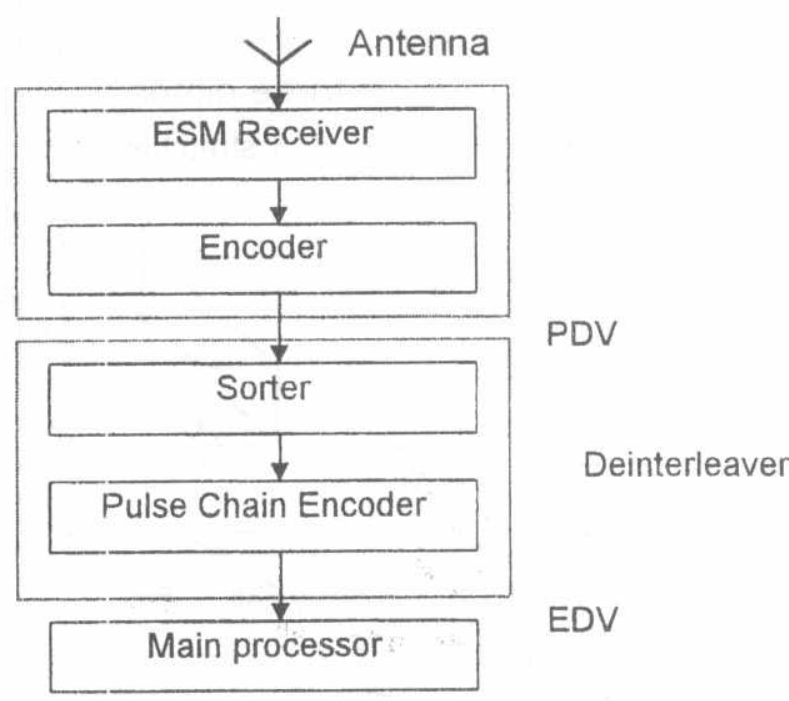

Fig.1: Basic layout of an automatic ESM system PDV: pulse descriptor vector EDV: Emitter descriptor vector

\begin{tabular}{|c|c|c|c|c|}
\hline $\begin{array}{c}\text { AOA } \\
{\left[M_{\text {AOA }} \text { bits }\right]}\end{array}$ & $\begin{array}{c}\mathrm{RF} \\
{\left[M_{R E} \text { bits }\right]}\end{array}$ & $\begin{array}{c}\text { PW } \\
{\left[M_{\text {PW }} \text { bits }\right]}\end{array}$ & $\begin{array}{c}\text { TOA } \\
{\left[M_{\text {TOA }} \text { bits }\right]}\end{array}$ & $\begin{array}{c}\text { PA } \\
{\left[M_{P A} \text { bits }\right]}\end{array}$ \\
\hline
\end{tabular}

Fig. 2. Representation of the information fields of a PDV

$M_{\text {parameter }}$ is the number of bits used to encode the parameter

\section{THE PRPOSE.D DEINTERLEAVING ALGORITHM}

The deinterleaver processor operates on the PDVs, which represents the monopulse data and attempts to identify pulse cells due to individual emitters. Generally, deinterleaving algorithms can be classified [5] as real-time (analog) algorithms and computer-basec ones. The second type is of interest here and it is applied after digital encoding of measured mono-pulse parameters. Computer-based algorithms are further classified on the basis of whether they use the parameters of more than one pulse as pulse repetition interval (PRI) or they use the parameters of single pulse such as AOA, RF, PW and PA. The first type of the computer based algorithms are denoted as the interval only deinterleaving algorithms while the other type is denoted as the multiple prarameter deinterleaving algorithms $[5,6,7]$. 
Our proposed deinterleaving aigorithm belongs to the last type and it is described as follows; suppose, an automatic ESM system receives the first pulse immediately after switching on. The AOA, RF, PW of the first pulse, are measured and used to define a unique point in a three dimensional space. The coordinates of this space are the AOA, PW and RF. The first cell (cell 1) is centered on this point, having a volume representing the domain of that cell. The length of the sides of this cell is equal to the maximum permissible mismatch in the measured parameter. The next received pulse will either fall into that domain or outside it. If it falls into the domain of call 1, that pulse will be labeled as also belonging to cell 1 , and the domain of cell 1 will be updated to reflect the center of population of pulses belonging to it. Subsequent pulses that don't fall into the domain of cell 1 create their own cells. Fig. 3 visualized the segregation of interleaved pulses in three dimensional space.

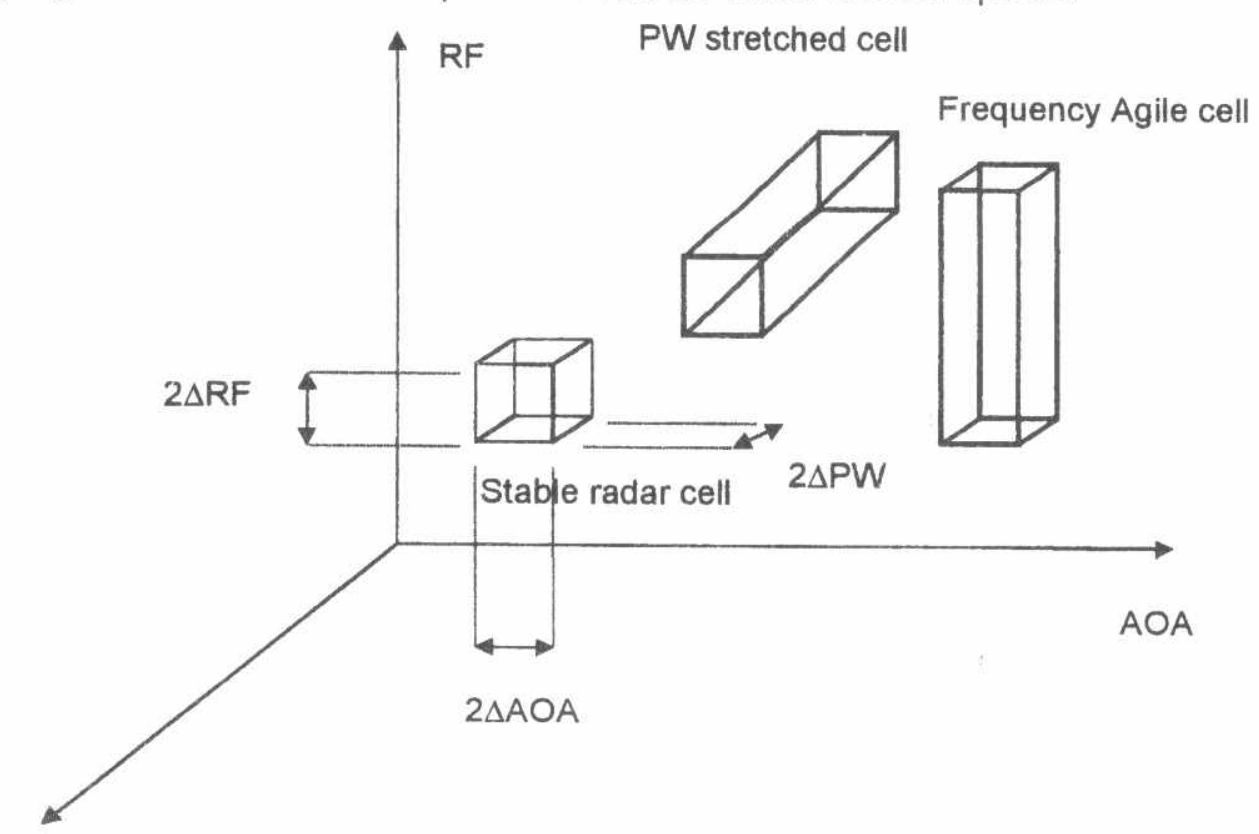

PW

Fig. 3. Three dimensional representation of segregation of interleaved pulse cells

It is important to know that the $i^{\text {th }}$ pulse falls in the domain of the $k^{\text {th }}$ cell depending on the value of the metric coefficient $M_{k, i}$ between cell $k$ and pulse $i[7]$, which is calculated from the following equation:

$$
M_{K, i}=\frac{\left(A O A_{k}-A O A_{i}\right)^{2}}{(2 \Delta A O A)^{2}}+\frac{\left(R F_{k}-R F_{i}\right)^{2}}{(2 \Delta R F)^{2}}+\frac{\left(P W_{k}-P W_{i}\right)^{2}}{(2 \Delta P W)^{2}}
$$

Where, $A O A_{k}$ is the average $A O A$ of the $k^{\text {th }}$ cell, $A O A_{i}$ is the $A O A$ of the $i^{\text {th }}$ pulse, $2 \triangle A O A$ is the maximum permissible mismatch in $A O A . R F_{k}$ is the average RF of the $k^{\text {th }}$ cell, $R F i$ is the RF of the $i^{\text {th }}$ pulse, $2 \Delta R F$ is the maximum permissible mismatch in RF. $P W_{k}$ is the average $P W$ of the $k^{\text {th }}$ cell, $P W_{i}$ is the $P W$ of the $i^{\text {th }}$ pulse and $2 \Delta P W$ is the maximum permissible mismatch in PW. It should be noted that the maximum permissible mismatch in any parameter is assumed to be double the root mean 
square error of measuring that parameter. From Chebyshev inequality [8] these maximum permissible mismatches guarantee a confidence level, not less than $75 \%$, of correctly attacining $i^{\text {th }}$ PDV to $\mathrm{k}^{\text {th }}$ radar cell.

In our proposed deinterleaving algorithm we enhanced the multiple parameter deinterleaving algorithm given in [7] by adding weights to the deinterleaving parameters in eqquation (1) and set a threshold value for the metric coefficient. Thus, the $i^{\text {th }}$ DV attached to the $k^{\text {th }}$ radar cell if its components satisfy the following inequalty:

$$
M_{k, i}<H
$$

Where, $H$ is the threshold value and $M_{k, i}^{\prime}$ is the modified mefric coefficient which is given by

$$
M_{K, i}=W_{A O A} \frac{\left(A C A_{k}-A O A_{i}\right)^{2}}{(2 \triangle A O A)^{2}}+W_{R F} \frac{\left(R F_{k}-R F_{i}\right)^{2}}{(2 \Delta R F)^{2}}+W_{P W} \frac{\left(P W_{k}-P W_{i}\right)^{2}}{(2 \Delta P W)^{2}}
$$

Where, $W_{A O A}, W_{R F}$ and $W_{P W}$ are the weights of $A O A, R F$ and $P W$ respectively. They are chosen such that

$$
W_{A O A}+W_{R F}+W_{P W}=1
$$

The AOA is considered as the most important deinterleaving parameter because of its stationarity $[1,2]$. The RF is chosen as the second deinterleaving parameter $[1,2]$. It should be ncted that frequency-agile radars could cause the apparent of large number of radar cells representing the same emitter. The PW is one of the unreliable parameter because of two reasons. The first reason is the time overlapping of radar pulses. The second is the effect of the multi-path problem, which distorts the pulse shape thus, the PW information is inconvenient for deinterleaving of radar pulses. From the above discussion It is evident that the domain of the generated radar cells can follow the variations in frequency and PW dimensions only but should be limited to the maximum permissible mismatch in $A O A$. Thus, the weight $W_{A O A}$ is taken between 0.985 and 1 while $W_{R F}$ and $W_{P W}$ are chosen less than or equal to 0.01 and 0.005 , respectively. Inside each deinterleaved radar cell, the difference in time of arrivals of the successive radar pulses belonging to this radar cell enables us to estimate the value of the PRI of this cell as described in $[5,6,7]$.

It is iniportant to know that the performance of the deinterleaver is much improved when it is preceded by a pre-buffer of size $\mathrm{K}$. The function of this pre-buffer is to store up to $\mathrm{K}$ of the arriving PDVs when the deinterleaver processor is busy. However, if a new PDV arrives at the deinterleaver processor when the pre-buffer is full, this PDV will be blocked. The blocking probability of the deinterleaver depends on the arrival rate of PDVs at the deinterleaver input, the comparison time inside the deinterleaver (the time needed to compare the input PDV with generated radar cells) and the size of the pre-buffer. It is shown [9] that, the deinterleaver processor can be represented as a queueing model. It is proved that, the blocking probability $\pi_{\kappa}$ of the deinterleaver is derived as: 


$$
\pi_{K} r_{0}=\pi_{K-1}-\sum_{j=1}^{K-1} \pi_{j} r_{K+1-j}
$$

Where, $\pi_{j}$ is the probability of existing $j$ PDVs inside the pre-buffer, and $r_{j}$ is the probability of receiving $\mathrm{j}$ PDVs at the input of the deinterleaver during processing of certain PDV.

It is clear that the number of bits used to generate the PDV will determine the value of the comparison time inside the deinterleaver. Thus, the longer the length of the PDV, the higher the blocking probability of the deinterleaver processor. So, if we desire to decrease the comparison time, the number of bits used to represent the PDV should be decreased, which means that the resolution and accuracy of measuring parameters of the intercepted pulse will be degraded. Thus, to obtain good measurement performance of each parameter of the intercepted pulse without increasing the blocking probability of the buffer we have two solutions. The first one is to increase the pre-buffer size and the second is to choose faster processor which decreases the comparison time.

The final effect of pulses lost due to blocking of the deinterleaver, is a wrong estimation of radars existing in the instantaneous view of the ESM receiver. Two common wrong decisions are usually encountered in the processing of the output of an ESM system. The first is the merging of two existing radars in the instantaneous view of the ESM receiver into one radar cell. The second is splitting of existing radar into two cells or more.

\section{COMPUTER SIMULATIONS}

A computer program is constructed using $\mathrm{C}^{++}$language to simulate the proposed deinterleaving algorithm. The main steps of the simulation program can be summarized as follows:

1- Simulation of the instantaneous view of an ESM system. We assume that certain number of radar emitters exist in the instantaneous view of an ESM system. We assume that these radars have low probability of intercept (LPI) and therefore have very low side lobe level ( $\mathrm{SLL}=-40 \mathrm{~dB}$ or below). The main lobes of the intercepted radars illuminate continuously the ESM system during relatively short observation interval. Also we consider the existence of both frequency agile and stable radars. Of course if the radar is frequency agile radar, we consider that its band of agility falls completely within the frequency range of the instantaneous view of ESM system.

2- Simulation of the service discipline inside the ESM receiver-encoder. We suppose that the service discipline inside the ESM receiver-encoder subsystem is the nonparalayzable counter service discipline[1,9]. Under non-paralayzable service discipline the ESM receiver-encoder is ready to process a new pulse as soon as the previous pulse is expired.

3- Performance evaluation of the suggested deinterleaver processor.

From this simulations we can evaluate the performance of the proposed deinterleaving algorithm as a function of the speed of the deinterleaver processor, the size of the pre-buffer in front of the deinterleaver and root mean square error of measuring the mono-pulse parameters. 
Fig. 4 and Fig. 5 show quantitatively the effect of the speed of the deinterleaver processor and the size of the pre-buffer on the blocking probability of the deinterleaver as a function of the input arrival rate of PDVs. Fig. 4 shows that for a fixed pre-buffer size and fixed average arrival rate of PDVs, the higher the speed of the deinterleaver processor, the lower the probability of blocking. Also, the probability of blocking is much reduced by increasing the size of the pre-buffer, for fixed speed of the processor, as shown in Fig. 5.

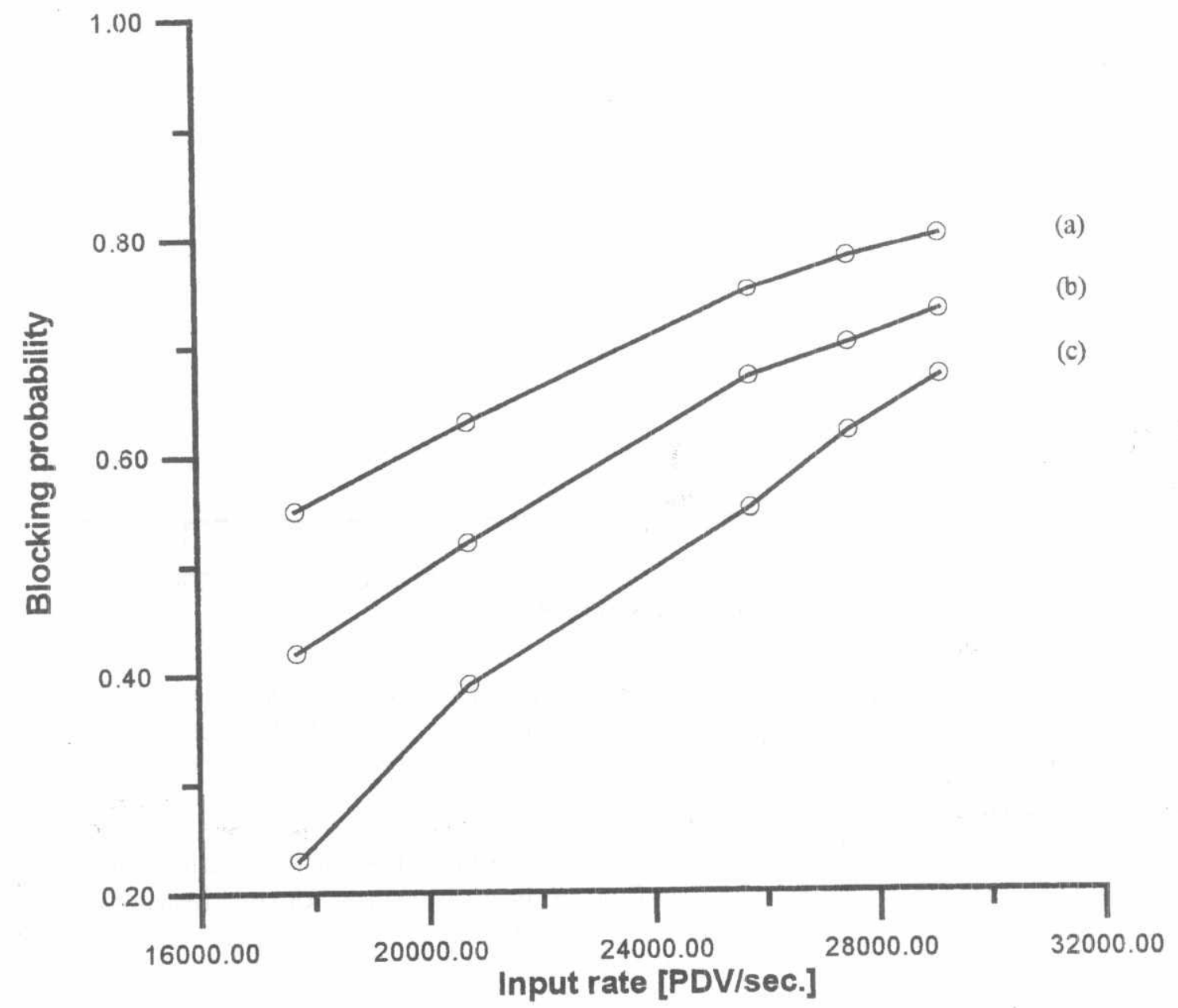

Fig. 4. Dependence of the blocking probability on arrival rate of PDVs, constant pre-buffer size $=5$ PDVs: (a) comparison time $=45 \mu$ sec, (b) comparison time $=35 \mu \mathrm{sec}$, (c) comparison time $=25 \mu$ sec. 


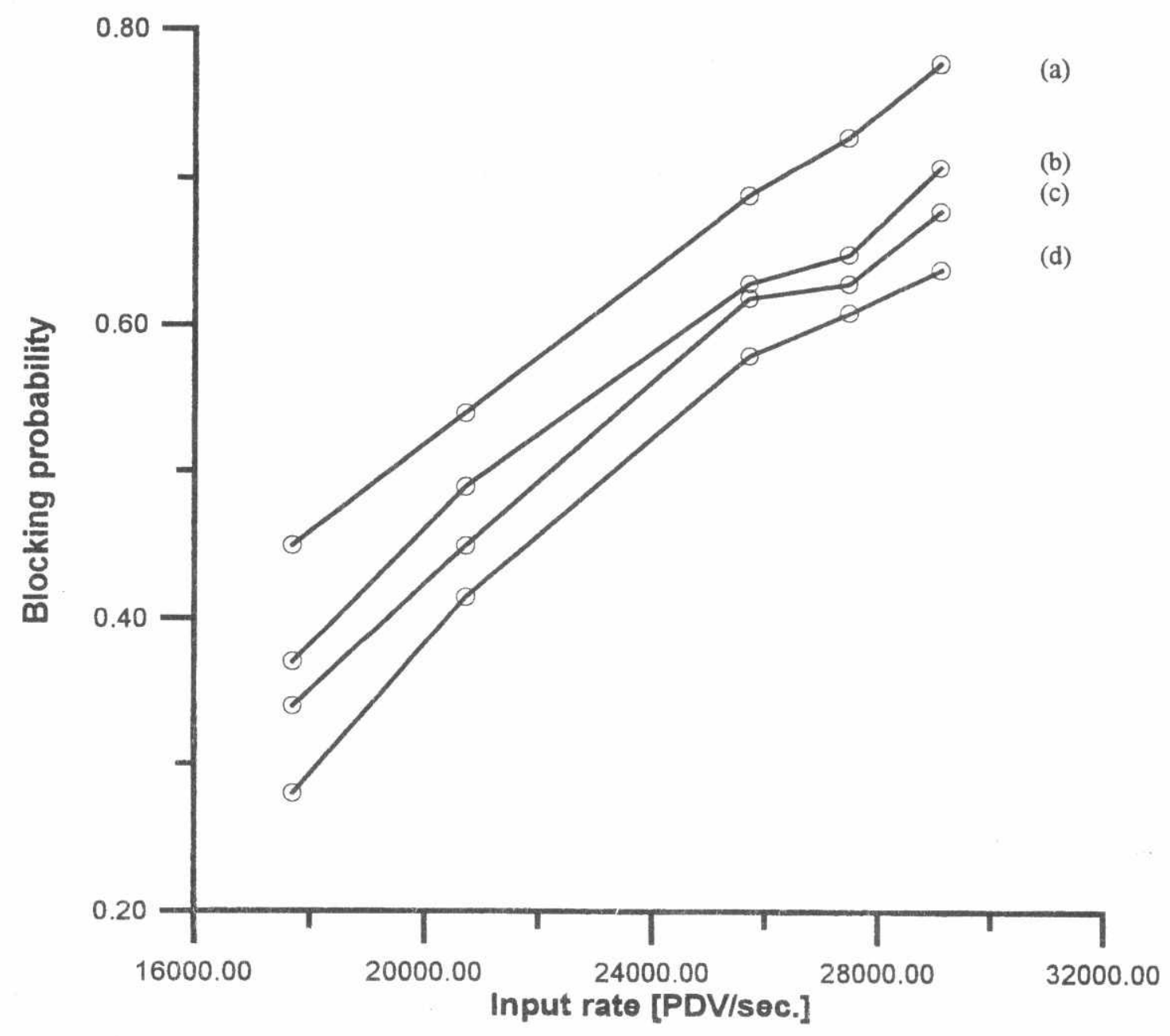

Fig. 5:Dependence of blocking probability on arrival rate, constant comparison time $=35 \mu \mathrm{sec}$ : (a) pre-buffer size = O PDVs, (b) pre-buffer size $:=20$, (c) pre-buffer size $=40$ PDVs, (d) pre-buffer size $=80$ PDVs

Now, we present the output of our simulation program in two study cases. In these two simulations, the metric coefficient is completely dependent on AOA because of its high stationarity. Thus, we will set $W_{A O A}=1, W_{R F}=W_{P W}=0$ in equation (4).

\section{CASE 1:}

The assumed number of radars in the environment: 7

The length of the otsservation time: $.035 \mathrm{sec}$.

The rate of radar pulses at the ESM receiver input $=30212 \mathrm{Pulse} / \mathrm{sec}$.

The total number of received pulses $=1057$ pulses

The rate of PD'Vs at the deinterleaver input $=25760 \mathrm{PDV} / \mathrm{sec}$.

$$
\mathrm{AOA}=2.5^{\circ}
$$

The comparison time: $.0000035 \mathrm{sec}$.

The pre-buffer size: 5

The blocking probability of the deinterleaver $=0 \%$

The number of generated cells: 7 
Table 1 and Table 2 present the parameters of the existing radars in the instantaneous view of ESM system and the parameters of the estimated radar cells respectively.

Table 1. Parameters of the radars contributing to the instantaneous view of the ESM receiver

\begin{tabular}{|c|c|c|c|c|c|c|}
\hline $\begin{array}{c}\text { Radar } \\
\text { Number }\end{array}$ & $\begin{array}{c}\text { AOA } \\
{[\text { Degree }]}\end{array}$ & $\begin{array}{c}\mathrm{RF} \\
{[\mathrm{M} \mathrm{Hz}]}\end{array}$ & $\begin{array}{c}\mathrm{PW} \\
{[\mu \mathrm{sec} .]}\end{array}$ & $\begin{array}{c}\text { PRF } \\
{[\mathrm{Hz}]}\end{array}$ & Agility & $\begin{array}{c}\text { Received } \\
\text { Pulses }\end{array}$ \\
\hline 1 & 59 & 6500 & 6.5 & 5500 & Agile & 192 \\
\hline 2 & 23 & 3500 & 3.5 & 5000 & Agile & 175 \\
\hline 3 & 32 & 4250 & 8 & 4750 & Agile & 166 \\
\hline 4 & 14 & 5750 & 2.5 & 4000 & Agile & 140 \\
\hline 5 & 41 & 5000 & 4.5 & 3500 & Agile & 122 \\
\hline 6 & 5 & 2000 & 1.5 & 3000 & Not agile & 105 \\
\hline 7 & 50 & 5750 & 5.5 & 4500 & Agile & 157 \\
\hline
\end{tabular}

Table 2. Estimated radar cells at the output of the deinterleaver processor

\begin{tabular}{|l|l|l|l|l|l|l|}
\hline $\begin{array}{l}\text { Radar } \\
\text { Cell }\end{array}$ & $\begin{array}{l}\text { AOA } \\
{[\text { Degree] }]}\end{array}$ & $\begin{array}{l}\text { RF } \\
{[\mathrm{M} \mathrm{Hz}]}\end{array}$ & $\begin{array}{l}\text { PW } \\
{[\mu \text { sec. }]}\end{array}$ & $\begin{array}{l}\text { PRF } \\
{[\mathrm{Hz}]}\end{array}$ & Agility & $\begin{array}{l}\text { Received } \\
\text { Pulses }\end{array}$ \\
\hline 1 & 60 & 6487 & 8 & 5525 & Agile & 177 \\
\hline 2 & 24 & 3492 & 4 & 4976 & Agile & 131 \\
\hline 3 & 33 & 4227 & 9 & 4762 & Agile & 148 \\
\hline 4 & 15 & 2742 & 4 & 4000 & Agile & 120 \\
\hline 5 & 42 & 4991 & 6 & 4000 & Agile & 104 \\
\hline 6 & 6 & 2014 & 3 & 2995 & Not agile & 44 \\
\hline 7 & 51 & 5731 & 7 & 4505 & Agile & 127 \\
\hline
\end{tabular}

As shown in Table 1 and Table 2, the output of our simulation program is seven radar cells. The parameters of radar cells from 1 to 7 match the same parameters of the actual radars in the instantaneous view of ESM receiver. We obtain this result because the difference in the AOA between radars in the instantaneous view of the ESM system is greater than double the root mean square error of measuring AOA.

\section{CASE 2:}

The assumed number of radars in the environment: 9

The length of the observation time: $035 \mathrm{sec}$.

The rate of radar pulses at the ESM receiver input $=34549 \mathrm{Pulse} / \mathrm{sec}$.

The total number of received pulses $=1209$ pulses

The rate of PDVs at the deinterleaver Input $=27410 \mathrm{PDV} / \mathrm{sec}$.

$$
\mathrm{AOA}=2.5^{\circ}
$$

The comparison time: $.00000035 \mathrm{sec}$.

The pre-buffer size: 5

The blocking probability of the deinterleaver $=8.23244 \%$

The number of generated cells: 9 
Table 3 and Table 4 present the parameters of the existing radars in the instantaneous view of ESM system and the parameters of the estimated radar cells respectively.

Table 3. Parameters of the radars contributing to the instantane ous view of the ESM receiver

\begin{tabular}{|l|l|l|l|l|l|l|}
\hline $\begin{array}{l}\text { Radar } \\
\text { Number }\end{array}$ & $\begin{array}{l}\text { AOA } \\
{[\text { Degree }]}\end{array}$ & $\begin{array}{l}\text { RF } \\
{[\mathrm{M} \mathrm{Hz}]}\end{array}$ & $\begin{array}{l}\mathrm{PW} \\
{[\mu \text { sec. }]}\end{array}$ & $\begin{array}{l}\text { PRF } \\
{[\mathrm{Hz}]}\end{array}$ & Agility & $\begin{array}{l}\text { Received } \\
\text { Pulses }\end{array}$ \\
\hline 1 & 59 & 6500 & 6.5 & 5500 & Agile & 192 \\
\hline 2 & 23 & 3500 & 3.5 & 5000 & Not agile & 175 \\
\hline 3 & 32 & 4250 & 8 & 4750 & Not agile & 166 \\
\hline 4 & 5 & 2000 & 1.5 & 3000 & Agile & 105 \\
\hline 5 & 41 & 5000 & 4.5 & 3500 & Agile & 122 \\
\hline 6 & 14 & 2750 & 2.5 & 4000 & Agile & 122 \\
\hline 7 & 50 & 5750 & 5.5 & 4500 & Agile & 157 \\
\hline 8 & 68 & 8000 & 4.5 & 1750 & Not agile & 61 \\
\hline 9 & 10 & 3000 & 7.5 & 2700 & Agile & 94 \\
\hline
\end{tabular}

Table 4. Estimated radar cells at the output of the deinterieaver processor

\begin{tabular}{|l|l|l|l|l|l|l|}
\hline $\begin{array}{l}\text { Radar } \\
\text { Cells }\end{array}$ & $\begin{array}{l}\text { AOA } \\
{[\text { Degree }]}\end{array}$ & $\begin{array}{l}\text { RF } \\
{[\mathrm{M} \mathrm{Hz}]}\end{array}$ & $\begin{array}{l}\text { PW } \\
{[\mu \text { sec. }]}\end{array}$ & $\begin{array}{l}\text { PRF } \\
{[\mathrm{Hz}]}\end{array}$ & Agility & $\begin{array}{l}\text { Received } \\
\text { Pulses }\end{array}$ \\
\hline 1 & 60 & 6485 & 8 & 5490 & Agile & 170 \\
\hline 2 & 24 & 3500 & 4 & 4996 & Not agile & 125 \\
\hline 3 & 33 & 4250 & 9 & 4735 & Not agile & 145 \\
\hline 4 & 7 & 2040 & 2.5 & 3085 & Agile & 138 \\
\hline 5 & 42 & 4975 & 5 & 3487 & Agile & 104 \\
\hline 6 & 13 & 2720 & 3 & 4060 & Agile & 162 \\
\hline 7 & 52 & 5710 & 6.5 & 4475 & Agile & 125 \\
\hline 8 & 67 & 7985 & 6 & 1735 & Not agile & 40 \\
\hline
\end{tabular}

As shown in Table 3 and Table 4, the output of our simulation program is eight radar cells. The parameters of radar cells from 1 to 8 match the same parameters of the actual radars in the instantaneous view of ESM receiver. Radar number 9 is missed because of splitting the received pulses from it into radar cell number 4 and 6 . It is clear that radar number 9 is missed because its AOA is within double the root rnean square error of $\mathrm{AOA}$ of radars number 4 and 6 .

Generally, the average values of the parameters inside each estimated cell are approximately matched to the exact parameters of the corresponding emitters. This is achieved by applying strict criteria to estimate the radar parameters such as PRF and agiiity inside each cell.

\section{CONCLUSION}

In this paper, there has been analyzed the automatic deinterleaving of intercepted radar pulses in dense emitter environments. The deinterleaver sorts the PD'Vs output from the receiver-encoder subsystem into separate radar cells 
according to the proposed deinterleaving algorithm. The proposed deinterleaving algorithm relied on $A O A, R F$ and PW components of the PDVs. Additional parameters such as PRI is derived through the analysis of each individual cell. The authors have constructed a computer program using $\mathrm{C}^{++}$Language to simulate automatic deinterleaving of intercepted radar pulses. The simulation results show that the blocking probability of the deinterleaver decreases as the size of the prebuffer in front of the deinterleaver increases at the same arrival rate of PDVs and the same processing speed of the deinterleaver. Also, the results shows that the blocking probability of the deinterleaver decreases as processing speed of the deinterleaver increases at the same arrival rate of PDVs and the same size of the pre-buifer in front of the deinterleaver. It has been also concluded that, the root mean square error of measuring the intercepted pulse parameters has a greater effect on the deinterleaving process.

\section{REFERENCES}

[1] CURTIS, S., INTRODUCTION TO ELECTRONIC WARFARE, Artech House, Inc.., (1986).

[2] DAVIES, C. L. and HOLLANDS, P., " Automatic processing for ESM, "IEE proc. F, Comm., Radar \& Signal Process, 129, (3), pp. 146-171, (1982).

[3] TSUI, J. B. MICROWAVE RECEIVERS with ELECTRONIC WARFARE APPLICATIONS, J. WILEY \& SONS, (1986).

[4] WILEY, R.G., THE INTERCEPTION OF RADAR SIGNALS, Artech House, Inc., (1982).

[5] MILOJEVIC, D. J. and POPOVIC, B. M., "Improved algorithm for deinterleaving of radar pulses," IEE Proc. F, Comm., Radar \& Signal Process., 139, (1), pp. 98104, (1992).

[6] MARDIA, H. K., "New techniques for the deinterleaving of repetitive sequence, " IEE. F, Comm. Radar \& signal Process., 136, (4), pp. 149-154, (1989).

[7] WILKINSON, D. R., and WATSON, A." Use of metric techniques in ESM data processing," IEE Proc. F, Comm., Radar F, Comm., Radar \& Signal Process., $132,(7)$, pp.121-125, (1985).

[8] FREDRIC de COULON, Signal Theory and Processing, Artech House, Inc., (1986).

[9] Abou-Bakr, H., "Analysis of the Queueing Behavior of automatic ESM System in Dense Emitter Environments," Master of Science Degree in Electrical Engineering, M. T. C., Cairo, Egypt, (1998). 